\title{
Labyrinthe
}

28 | 2007 (3)

Des Juifs contre l'émancipation

\section{Un Aufklärer entre deux feux : Moses \\ Mendelssohn (1729-1786)}

\section{Franck Lemonde}

\section{(2) OpenEdition \\ 1 Journals}

Édition électronique

URL : http://journals.openedition.org/labyrinthe/2873

DOI : $10.4000 /$ labyrinthe.2873

ISSN : 1950-6031

Éditeur

Hermann

Édition imprimée

Date de publication : 21 décembre 2007

Pagination : 53-61

ISBN : 978-2-9526131-5-6

\section{Référence électronique}

Franck Lemonde, «Un Aufklärer entre deux feux : Moses Mendelssohn (1729-1786) », Labyrinthe [En

ligne], 28 | 2007 (3), mis en ligne le 01 octobre 2009, consulté le 21 avril 2019. URL : http:// journals.openedition.org/labyrinthe/2873; DOI : 10.4000/labyrinthe.2873

Propriété intellectuelle 


\title{
UN AUFKLÄRER ENTRE DEUX FEUX : MOSES MENDELSSOHN (1729-1786)
}

\author{
Franck LEMONDE \\ franck_lemonde@yahoo.fr
}

\begin{abstract}
Devant Ton intelligence, les princes ont eu le souffle coupé, les vieillards se sont redressés et se sont levés pour savourer le plein agrément de Tes paroles, qui unissent des formules pleines de caractère et des mots pénétrants, Ta gloire est toujours sur nos lèvres, Tu es notre parure, car Tu nous as soulagés de l'opprobre de tous ces peuples, qui jour après jour nous dénigraient de la sorte: honte à toi, mépris sur toi, peuple à la langue lourde, mouton тиеt au langage répugnant et sans entendement! Mais maintenant il va être connu parmi les peuples devant nos yeux. Car Israël n'est pas orphelin, et ne tombe pas sous leur mêlée et leur cohue. Ils Te voient, ils Te considèrent, ils Te contemplent : est-ce Lui, l'homme qui se glorifie du saint Israël? Qui se consacre à Dieu et est appelé du nom d'Israël? Sur Lui souffle l'esprit de la sagesse et de la compréhension; au regard de leur compréhension, une intelligence double. C'est Toi que la raison a élu pour Te placer en porte-drapeau des peuples, et c'est Toi, l'élu, qui donne au peuple élu son bon renom, son éclat et sa gloire' ${ }^{1}$
\end{abstract}

C'est ainsi que la communauté juive de Dresde, sous la plume du rabbin Samuel Halberstadt, s'adressait pour faire une requête à celui que la tradition rationaliste a plutôt tendance à appeler «le Socrate de Berlin». Rationaliste, Mendelssohn le fut sans aucun doute; disciple de Socrate également - n'avait-il pas fait son entrée dans le concert philosophique européen en 1767 par une retentissante «reprise» en termes modernes du Phédon de Platon, aussitôt traduite en une dizaine de langues? Cet aspect-là de l'Aufklärer (homme des Lumières) n'est pas

1. Lettre de la communauté juive de Dresde à Mendelssohn, novembre 1777 (mar-'hechvan 5537), traduite d'après la traduction allemande du texte hébreu donnée dans Moses Mendelssohn, Gesammelte Schriften [à partir d'ici GS] 20, 2, Stuttgart, Jubiläumausgabe, Friedrich Fromman Verlag, 1994, p. 374. 
le plus méconnu en France; Mirabeau avait été le premier à rendre hommage à celui qui, selon ses dires, s'était extrait du «bourbier talmudique » (sic) dans lequel il avait été élevé pour parvenir aux lumières de la raison universelle et contribuer si brillamment à leur diffusion ${ }^{1}$. Mais cette version «laïciste ${ }^{2} »$ est une simplification qu'il ne serait peut-être pas inutile de déjouer en revenant sur la relation beaucoup plus subtile que Mendelssohn a entretenue avec la foi de ses pères et ses contemporains coreligionnaires.

De fait, le combat mené par Mendelssohn pour la reconnaissance des droits des Juifs en Allemagne et en Europe (qu'il n'a jamais à ma connaissance intitulée «émancipation» mais, comme nous le verrons plus loin, «admission civile», bürgerliche Aufnahme), s'il se faisait, dans l'esprit de son fidèle ami Lessing, au nom de la commune raison, de la commune appartenance à l'humanité des Juifs et des chrétiens, ce combat ne s'est jamais accompagné d'un abandon de cette identité religieuse, ou d'un appel à l'abandon comme condition de leur admission. Ses disciples, comme David Friedländer ${ }^{3}$, et ses propres enfants, comme la future Dorothea Schlegel ${ }^{4}$, s'en chargeront. Je voudrais donc cerner l'ambivalence du rôle de Mendelssohn, et sans dénier son indubitable influence dans le processus d'assimilation-dissolution, rendre un peu plus visible son refus indéfectible de toute concession face aux pressions chrétiennes qu'il eut régulièrement à subir. J'aimerais aussi mieux saisir son rôle d'intercesseur pour la communauté juive d'Allemagne (comme en témoigne la lettre citée plus haut), et comment ce rôle fut perçu par les Juifs eux-mêmes - entre la reconnaissance pour le «sauveur» et la suspicion pour le «novateur». Entre deux feux, donc, car Mendelssohn dut constamment justifier sa position des deux

\footnotetext{
1. Le texte de Mirabeau s'intitule Sur Moses Mendelssohn et la Réforme politique des Juifs. Il peut se lire dans son intégralité, en peu de temps et avec plaisir sur www.gallica.bnf.fr.

2. J'entends par «laïcisme» l'idéologie qui identifie la religion à la superstition et la science à la vérité. 3. «Le plus puissant avantage dont les Juifs pourraient tirer parti serait d'éloigner de plus en plus de leurs cœurs leur nostalgie du Messie et de Jérusalem, à l'instar de la raison qui rejette sans cesse cette espérance comme chimérique.», David Friedländer, Sendschreiben einiger jüdischer Hausväter, cité par Hannah Arendt, La Tradition cachée, trad. Sylvie Courtine-Delamy, Paris, Christian Bourgois, 1987, p. 23.

4. Dont Hannah Arendt dira: «Elle ne s'assimile pas au romantisme, mais à Schlegel; elle ne se convertit pas au catholicisme, mais à la foi schlégélienne», ibidem, p. 41. Rappelons que le compositeur Felix Mendelssohn est le petit-fils du philosophe et le fils d'Abraham Mendelssohn, banquier. Il a notamment tiré J. S. Bach de l'oubli et mis en musique les Psaumes de David ainsi que les chœurs d'Antigone.
} 


\section{Un «Aufklärer» entre deux feux: Moses Mendelssohn}

côtés (qui eux-mêmes ne formaient pas deux blocs monolithiques); ce pourquoi on lui fera grief d'avoir contribué à une certaine «schizophrénie» juive dans la modernité 1 . Pour reprendre l'expression de Jacques Rancière, j' aimerais voir en Mendelssohn un «schizophrène en bonne santé ${ }^{»}$.

Dès la publication de son Phédon, Mendelssohn éprouva la crainte de recevoir des critiques sévères du côté rabbinique. Il s'en confie à son ami poète Hartog Wessely qui voulait traduire le traité en hébreu:

Je recherche, j'apporte des preuves et je donne des exemples sur un sujet [l'immortalité de l'âme] dont la vérité, aux yeux de tout homme qui se nomme israélite, ne peut être mise en doute, car elle est une branche des fondements de notre croyance sacrée ${ }^{3}$.

\section{À quoi Wessely, en bon maïmonidien, répondra:}

Jamais il ne fut interdit de mettre au clair (klarlegen) les objets de croyance et d'opinion par des preuves rationnelles; tous les mots de la vérité sont les enfants du Dieu unique; la croyance et la Torah et la fidèle tradition, la nature et la recherche avec la droite raison, toutes sont données par un berger unique, par le vrai Dieu. Et vive celui qui renforce les principes de la croyance par des preuves rationnelles ${ }^{4}$ !

Le fait est que ce Phédon ne suscitera pas le courroux, mais l'indifférence polie de la plupart des rabbins. Son retentissement allait par contre inciter un jeune théologien suisse, le diacre Lavater, à vouloir convertir violemment cet intrus dans la république des lettres, en sommant cet amateur de preuves rationnelles de prouver rationnellement et publiquement la fausseté du dogme de la divinité du Christ - en vain, car Mendelssohn répliquera que la religion, et notamment la sienne, n'a pas à perdre de temps ni de talent dans la controverse:

\footnotetext{
1. Ainsi Yirmiyahu Yovel dans son article assez amer intitulé «Mendelssohn Projekt: Vier Herausforderungen », Die philosophische Aktualität der jüdischen Tradition, Francfort, Suhrkamp, 2000, p. 331-350.

2. Ce concept est étayé dans Politique de la littérature, Paris, Galilée, 2007. La notion de schizophrénie, décidément importante pour ce qui nous occupe ici, se trouve aussi dans l'entretien avec Pierre Birnbaum publié dans la présente livraison de Labyrinthe.

3. Lettre à Hartog Wessely, septembre 1768 (elul 5528), GS, 20, 2, p. 160 sqq.

4. Lettre de Hartog Wessely à Moses Mendelssohn (elul 5528), ibidem, p. 162 sqq.
} 
L'opinion méprisante que l'on se fait d'un Juif, j'aimerais pouvoir la réfuter par la vertu (Tugend) et non par les écrits polémiques (Streitschriften). Ma religion, ma philosophie et mon état dans la vie civile me fournissent les motifs les plus importants d'éviter toute querelle religieuse (Religionsstreitigkeiten) et de parler, dans les écrits publics, seulement des vérités qui doivent être d'égale importance pour toutes les religions ${ }^{1}$.

Le coup porté ne fut toutefois pas sans conséquence sur l'énergie spirituelle momentanément brisée du maskil. Il n'est pas indifférent que celui-ci ait choisi pour se ressourcer (car il avait «perdu la capacité de méditer ${ }^{2} »$, selon ses dires) de traduire en allemand les Psaumes de David, puis le Pentateuque. Ce geste peut-être interprété comme un retour aux sources, mais ne marque-t-il pas également un écart par rapport à la sacralisation de la langue de la Torah ${ }^{3}$ ? Ayant reçu l'approbation du grand rabbin de Berlin Hirschel Lewin mais les foudres du grand rabbin d'Altona Raphaël Cohen, le voilà contraint de s'expliquer sur la légitimité de ses intentions: dans une lettre à son ami rabbin Avigdor Levi, il affirme avoir voulu mettre une édition bilingue, fiable et parsemée de références aux commentaires canoniques, «à l'usage des enfants d'Israël qui cherchent un commentaire des versets et leur traduction en langue allemande dans les livres qui égarent (irrefiihrend) les nations », afin de «renforcer l'enseignement du Seigneur et tenir à l'écart (fernhalten) les enfants d'Israël des produits étrangers (fremden Erzeugnissen) qu'ils ont utilisés jusqu'à présent ${ }^{4} \gg$. Le ton est remarquablement différent dans une lettre à son ami diplomate danois August Hennings quasi contemporaine: certes, il s'agissait bien de donner «à

1. C'est moi qui souligne. Je traduis à partir de «Schreiben an den Herrn Diakonus Lavater in Zürich», dans Moses Mendelssohn, Schriften über Religion und Aufklärung, éd. Martina Thom, Berlin, Union Verlag, 1989, p. 314.

2. Lettre à August Hennings du 29 juillet 1779, GS, 12, 2 p. 149.

3. Je n'ignore plus que le Talmud (Megilah, $8 \mathrm{~b}$ et $9 \mathrm{a}-\mathrm{b}$ ) ne considère nullement la traduction comme une profanation, sauf pour les séquences de la Bible consacrées à l'usage rituel et insérées dans les objets de culte (tefilin et mezuzot). La seule condition requise pour qu'une traduction maintienne la sacralité de l'original est l'écriture en caractères hébraïques, ce que Mendelssohn ne manquera pas de respecter (à la différence de son ami éditeur Friedrich Nicolai en 1780 et, plus récemment, de la Jüdischer Verlag de Berlin...). Emmanuel Levinas synthétise remarquablement cette question dans «La traduction de l'écriture», Israël, le Judaïsme et l'Europe, données et débats, Paris, Gallimard, 1984, p. 336-362. Je crois pouvoir maintenir toutefois que ce choix de traduire, sans profaner ce qu'il s'approprie, ouvre la voie à sa désacralisation.

4. Je traduis la traduction allemande de la lettre à Avigdor Levi du 25 mai 1779 (10 sivan 5539), GS, 20, 2 p. 371-372. 
ses enfants et à une portion considérable de son peuple [...] une meilleure traduction et interprétation des livres sacrés qu'ils n'avaient eue jusqu'alors», mais, cette fois, car «ceci est le premier pas vers la culture (Cultur), dont ma nation, hélas! est si éloignée qu'on en viendrait presque à désespérer de la possibilité d'une amélioration ${ }^{1}$. Avec qui était-il le plus sincère ? La première lettre ayant été écrite en hébreu et la seconde en allemand, je préfère suspendre mon jugement ${ }^{2}$.

Le fait est que le crédit grandissant dont Mendelssohn bénéficia dans toute l'Allemagne cultivée s'accompagna, nous l'avons aperçu, d'un grand prestige dans la communauté juive. Cela toutefois au prix d'un certain malentendu:

Les jeunes hommes juifs se pressaient chez lui, comme on le faisait chez un rabbin célèbre [...]. Les sophismes des querelles talmudiques, les détails futiles et insignifiants des lois rituelles étaient déjà devenus l'objet de sarcasmes dans la vie de tous les jours, néanmoins, en présence de Mendelssohn, on se retenait de les évoquer, sérieusement ou pour plaisanter ${ }^{3}$.

Au moment même où il devenait ce «rabbin laïc» donnant accès aux 'hokhmot (les sagesses traditionnelles) à une génération d'Aufklärer radicaux, qui aspiraient à un savoir universel contre les hiérarchies disciplinaires traditionnelles privilégiant les textes sacrés ${ }^{4}$, Mendelssohn réaffirme son attachement à la tradition, et ce dans ses aspects les plus stricts et les plus minutieux, en rédigeant en 1778 à la demande du grand rabbin de Berlin une version synthétique, mais sans l'ombre d'une réforme, des lois rituelles juives concernant le mariage, l'héritage et la propriété. Acte ambigu dans ses conséquences car en voulant clarifier le droit juif pour les juristes allemands, il inaugure de

1. Lettre à August Hennings du 29 juillet 1779, GS, 12, 2, p. 149.

2. Le thème de la «double parole» (Doppelzüngigkeit) est récurrent dans les calomnies nazies dont Mendelssohn fut à titre posthume victime, tout comme, plus généralement, dans la tératologie antisémite.

3. David Friedländer, Fragmente, 1819, cité dans Stephen Tree, Moses Mendelssohn, Hambourg, Rowohlt, 2007, p. 78.

4. Lisons le témoignage sarcastique de Salomon Maïmon (1753-1800) dans son autobiographie: alors qu'il avait délaissé le Talmud pour lire en cachette des livres d'astronomie, son père rabbin l'y surprend et l'en dissuade provisoirement: «Qui connaît le Talmud connait toutes choses» (Lebensgeschichte, Francfort, Jüdischer Verlag, 1995). 
fait l'intervention du droit allemand dans la juridiction juive: on retrouve la problématique selon laquelle la sortie du ghetto politiquement libératrice implique une indéniable perte de l'autonomie culturelle.

Cette tension dialectique sera l'objet du chef-d'œuvre de philosophie politique de Mendelssohn, Jérusalem, ou sur le pouvoir religieux et le judaïsme, paru en 1782 après une nouvelle attaque venue du côté chrétien, cette fois sous la plume d'un ancien admirateur, August Cranz. Rappelons les circonstances: Mendelssohn avait écrit une préface à une traduction allemande (publiée par Friedrich Nicolaï) du fameux Salut des Juifs de Manasseh ben Israel, ce rabbin hollandais qui avait demandé (avec succès) à Cromwell la réadmission de son peuple en Angleterre ${ }^{1}$. Dans cette préface, Mendelssohn commençait par bénir la «toute clémente Providence» de l'avoir fait naître à une époque où, pour la première fois, ce n'est pas le parti opprimé qui cherche à «se sauver lui-même», mais le parti oppresseur, ou plutôt une minorité en son sein, qui se défait de sa supériorité («sich seiner Ueberlegenheit entaüssern») en réclamant l'extension universelle des droits dont il bénéficie ${ }^{2}$. La question de la tolérance n'avait été auparavant posée qu'entre les diverses branches du christianisme (ainsi le traité de Westphalie en 1648): quant aux païens, Juifs, mahométans et naturalistes, on les supportait («dulden»), on ne les tolérait pas. Autrement dit, on ne les laissait pas prendre part à tous les droits et devoirs de l'humanité. Mais les Lumières ont leur part d'ombre: contrairement à «l'époque superstitieuse» (sic) où l'on accusait les Juifs de crimes rituels, arts secrets, sorcellerie etc., «l'époque de la culture et du progrès » les accuse précisément de superstition, de brutalité et d'arriération. Des êtres aussi peu policés ne peuvent assurément se montrer utiles à la cité - et ne méritent ainsi que d'être expulsés «du giron maternel de l'État». Comme les efforts pour convertir les Juifs au christianisme ont été vains, on a estimé vain tout effort de les accueillir dans la communauté civile, les privant ainsi d'accès à la culture universelle, arts, sciences et métiers utiles. Et l'on fait «de ce manque de culture le fondement du renforcement de [leur] oppression». «On [leur] lie les mains, résume Mendelssohn, et on [leur] reproche de ne pas [s'] en ser-

1. Dont ils avaient été expulsés en 1290 sous Edouard I ${ }^{\text {er. }}$.

2. Je m'inspire de la Vorrede zu Manasseh ben Israëls Rettung der Jude placée avant Jerusalem, über das Judentum und die religiöse Macht dans sa dernière édition allemande, Bielefeld, Aisthesis Verlag, 2001, p. 8-30. 


\section{Un «Aufklärer» entre deux feux: Moses Mendelssohn}

$\operatorname{vir}^{1}$.» Non content d'avoir condamné toute forme d'exclusion pour la religion, Mendelssohn concluait sa préface en condamnant résolument toute forme d'exclusion par la religion - ainsi, explicitement, le 'herem (excommunication). C'est à ma connaissance l'unique réelle innovation proposée par Mendelssohn dans la législation juive². Outre la colère de certains rabbins, Mendelssohn s'attira alors la suspicion de certains chrétiens comme Cranz:

Comment pouvez-vous, mon cher Mendelssohn, vous entêter dans la foi de vos pères et, en enlevant ses fondements, renverser tout le bâtiment, si vous critiquez le droit canon (Kirchenrecht) donné à Moïse par révélation divine ${ }^{3}$ ?

La réponse fut donnée en 150 pages de philosophie politique très denses, agrémentées d'une réflexion profonde sur, entre autres, la distinction des vérités historiques et des vérités éternelles, le prestige du narrateur dans l'élaboration de la crédibilité ${ }^{4}$, l'historicité et la métaphoricité du langage ${ }^{5}$, ainsi qu'une étonnante critique de la théorie du progrès linéaire à laquelle le philosophe oppose une description des oscillations aléatoires de l'espèce humaine:

On se représente la chose collective, l'espèce humaine, comme une personne singulière, et l'on croit que la Providence l'a pour ainsi dire envoyée ici-bas à l'école, pour être élevée de l'enfance à l'âge d'homme. En fait c'est comme si l'espèce humaine était à tous les siècles, si la métaphore doit valoir, enfant, homme et vieillard. [...] La progression (Fortgang) vaut pour l'homme individuel, à qui la Providence a accordé de passer une partie de son éternité ici sur terre. Chacun va son propre chemin à travers la vie. [...] Mais que le tout également, l'humanité ici-bas, dans

\section{Ibidem, p. 11.}

2. Sachant que le 'herem était déjà de moins en moins pratiqué à l'époque en Allemagne. J'ignore ce qu'il en était en Europe de l'Est.

3. August Cranz, «Das Forschen nach Licht und Recht in einem Schreiben an Herrn Moses Mendelssohn», cité dans Stephen Tree, op. cit., p. 112.

4. Fondé sur le besoin naturel de présupposer comme vraies des observations auxquelles l'homme n'a pas pris part. L'autorité est donc requise pour la transmission des vérités historiques, mais tout sujet pensant, et volontaire pour penser en acte, a un accès direct à la contemplation des vérités éternelles. Cf. Jerusalem, op. cit., p. 86-89.

5. Je résume: les mots étant des métaphores des affects, les affects changeant selon les époques et les individus, les mêmes mots changent de sens selon les époques et les individus. Op. cit, p. 102 sqq. 
la suite des temps, se porte toujours vers l'avant, et doive se parfaire (sich vervollkommen), cela ne me semble pas avoir été le but de la Providence. [...] Vous ne trouverez pas, au point de vue de l'ensemble de l'espèce humaine, de progrès (Fortschritt) continu dans la formation qui se rapprocherait sans cesse de la perfection. Bien plus, nous voyons l'espèce humaine dans son ensemble faire de petites oscillations (kleine Schwingungen), et elle n'a jamais fait quelques pas vers l'avant sans aussitôt après, à vitesse redoublée, revenir à son état antérieur [...]. L'homme avance; mais l'humanité va et vient (schwankt) perpétuellement entre des bornes fixées, de haut en bas, et maintient, considérée dans son ensemble, dans toutes les périodes du temps, à peu près le même degré de moralité, la même mesure de religion et d'irreligion, de vertu et de vice, de béatitude et de misère ${ }^{1}$.

Preuve, s'il en était besoin, que Mendelssohn n'est pas tout à fait un Aufklärer "comme les autres» (à supposer d'ailleurs que cette chose existe). Mais surtout, pour en revenir à notre sujet, loin de céder à l'injonction de conversion, Mendelssohn réaffirme son entêtement en rappelant que le judaïsme ne prescrit aucune croyance à un dogme, mais des conduites et des actions, les mitzvot, sur l'inamovibilité desquelles il ne reviendra pas. Le 'herem n'en est pas moins à ses yeux une aberration car la religion, et toute religion, n'est vouée qu'à l'édification («Erbauung») et à l'amélioration spirituelle de ses sujets: exclure en est donc exclu, et cette pratique n'a de sens que dans le cadre du pouvoir temporel. «Lecteur, quelle que soit l'église extérieure, la synagogue ou la mosquée à laquelle tu adhères! demande-toi donc si tu ne rencontreras pas plus de vraie religion dans la masse des bannis que dans la masse infiniment plus grande de leurs bannisseurs ${ }^{2}$ !» Parallèlement à ces envolées et plus sobrement, Mendelssohn déduit du caractère uniquement législatif de la révélation l'éminente rationalité du judaïsme. Point de mystères, point de paradoxes de la foi défiant la

1. Jerusalem, op. cit, p. 93 Ceci est ma traduction. Il existe une version française de Jerusalem écrite par Dominique Bourel (Paris, Les Presses d'Aujourd'hui, 1982, rééd. Paris, Gallimard, 2007). Malgré tous ses mérites, et notamment celui d'exister, elle contient des inexactitudes importantes. Je souhaite y remédier, avec mes faillibles moyens, dans une anthologie de la pensée politique judéoallemande que j'intitulerai probablement Politique des Juifs allemands, de Moses Mendessohn à Ernst Bloch. Cet article en est le premier maillon.

2. Jerusalem, op. cit, p. 134. 
raison, la Torah n'est qu'un guide de sagesse qui laisse la place libre à la réflexion et à l'investigation scientifique'.

Mendelssohn continuera d'ailleurs à philosopher jusqu'à la fin de ses jours, produisant notamment à son crépuscule, en 1785, des Heures matinales (Morgenstunden) dans lesquelles il renouvelle la démarche rationaliste du Phédon en l'appliquant cette fois aux preuves de l'existence de $\mathrm{Dieu}^{2}$. Ce Dieu est commun à toutes les religions, mais la déclaration de son existence n'est pas censé en faire fusionner les différentes versions, dont aucune, selon la fable de l'anneau dans Nathan le Sage de Lessing, ne peut prétendre être «la bonne» (puisque l'original est perdu). L'unification des croyances n'est pas la tolérance ${ }^{3}$ : celle-ci est bien plutôt le maintien de la singularité propre à chaque spiritualité dans le partage de la commune rationalité. C'est en ce sens que le Socrate de Berlin, loin d'être le fossoyeur de l'identité religieuse juive européenne, est plutôt l'instigateur d'une redéfinition moderne du judaïsme, telle que Hermann Cohen la rassemblera sous le titre de «religion de la raison ${ }^{4} »$.

\footnotetext{
1. À peu près à la même époque, Voltaire, dans son Essai sur les mœeurs, puis Goethe, dans ses remarques en annexe à ce qu'on pourrait traduire le Divan occidoriental (Das West-östlicher Divan), dirent la même chose de l'Islam. Cette idée banale au XVIII ${ }^{\mathrm{e}}$ siècle est fort bien analysée par Maxime Rodinson, membre éminent de l'Union rationaliste, dans son nullement désuet Marxisme et monde musulman, Paris, Le Seuil, 1972, p. 95 sqq.

2. Lettre à Raphaël Levi, fin 1767, GS, 20,2, Cet ouvrage, écrit dans une langue remarquablement limpide, est la meilleure marque que Mendelssohn métaphysicien a tenu le programme d'écriture qu'il s'était fixé plus jeune: «Nous devons parler la langue des phénomènes (die Sprache der Erscheinungen), car c'est en celle-ci que nous nous comprenons le plus sûrement les uns les autres. [...] Ainsi les preuves et les motifs rationnels doivent pouvoir être traduits de la langue des phénomènes dans la langue de la vérité.» Lettre à Raphaël Levi, fin 1767, GS, 20, 2, p. 153 sqq. Emmanuel Kant, qui tenait Mendelssohn en haute estime, mais qui avait d'autres égards que celui-ci pour ses lecteurs, avait cru dans la langue plus opaque de la Critique de la raison pure (1781) avoir condamné pour toujours au délire toute entreprise de preuve rationnelle de l'immortalité de l'âme ou de l'existence de Dieu. Impressionné mais pas découragé par les intimidations du tribunal de la critique, Mendelssohn tenta sans doute de démontrer dans les Heures matinales, outre l'existence de Dieu, la simple possibilité de garder son innocence métaphysique après ce «verdict» auto-proclamé irrévocable.

3. Jerusalem, op. cit., p. 134.

4. Pour reprendre le titre du livre qu'il publia en 1919 (Religion der Vernunft aus den Quellen des Judentums, traduction française Religion de la raison tirée des sources du judaïsme, Paris, PUF, 1994).
} 